\title{
Kinetic Theory of the Langevin Saturation in Dilute Solution of Dipolar, Symmetric-Top Molecules in Spherical Solvents
}

\author{
W. Alexiewicz* And K. Grygiel \\ Nonlinear Optics Division, Institute of Physics \\ Adam Mickiewicz University \\ Umultowska 85, 61-614 Poznań, Poland \\ (Received May 9, 2008)
}

\begin{abstract}
Assuming the low molecular reorientation approximation, the formulae for the third-order electric polarization induced in liquids composed of rigid noninteracting dipolar, symmetric-top molecules in spherical solvents were derived. Our medium is acted on by a strong external dc bias electric field superimposed on a weak ac electric field, and the classical SmoluchowskiDebye equation for rotational diffusion of the symmetric-top molecules is applied. In order to highlight the influence of the anisotropy of rotational diffusion tensor components and the orientation of permanent dipole moment of the molecule on the complex linear and nonlinear electric susceptibilities, we present three-dimensional plots of the linear and nonlinear dispersion and absorption spectra, for different values of the frequency of ac electric field.
\end{abstract}

PACS numbers: 05.40.Jc, 77.22.-d, 77.22.Gm

\section{Introduction}

Linear and nonlinear dielectric relaxation phenomena in molecular liquids, although discovered many years ago, are still the basis for wide experimental and theoretical studies. The Smoluchowski-Debye model of rotational diffusion of noninteracting, spherical-top molecules in solutions has been successfully applied in description of linear dielectric relaxation [1-3], the optical Kerr effect [4-6], the light scattering $[7,8]$, various phenomena connected with the third-order electric polarization in liquids [9-11] and especially the nonlinear dielectric effect (NDE) measured at the frequency of the probe field [12-18]. This last effect, named the Langevin saturation, is characterized by a negative contribution to the nonlinear electric susceptibility $\chi(-\omega ; \omega, 0,0)$, due to a decrease in the potential energy of the polar molecules in dc electric field.

${ }^{*}$ corresponding author; e-mail: walex@amu.edu.pl 
The Smoluchowski-Debye rotational relaxation theory has been extended to non-linear effects by Kielich and his co-workers [9-11], and applied to describe the dispersional and absorptional behavior of the third-order electric polarization induced in liquids consisting of the spherical-top molecule by external reorienting electric fields. The nonlinear-Langevin type relaxation was succesfully measured, as the nonlinear dielectric effect on the frequency of the probe field, by De Smet, Hellemans, Jadżyn, Kędziora and co-workers in many papers [14-18]. The Smoluchowski-Debye equation for the rotational diffusion of symmetric-top molecules was used by Morita and Watanabe in description of optical birefringence $[19,20]$, by Dejardin in evaluation of the nonlinear dielectric response [21], and also by one of us [22]. An elegant solution of the Smoluchowski-Debye equation of asymmetric-top molecules was given by Kalmykov [23]. An excellent and extensive review of dielectric relaxation phenomena in liquids was given by Coffey [24].

The anomalous rotational diffusion model, given recently by Coffey, Kalmykov, Dejardin, Jadżyn and co-workers [25-27], permits, in contrast to the Smoluchowski-Debye approach, investigation of molecular dynamics of the non-Markovian type, and opens wide applications in investigation of dynamic phenomena in liquids. This "anomalous rotational diffusion approach" is based on the non-Markovian equation with non-integer time differential operator and, as an elegant extension both of the Smoluchowski-Debye model and the Cole-Davidson results, includes these two types of relaxation [27]. These modern theories are in good agreement with the ac Kerr effect response experiments, performed on a dilute solution of poly(3-hexylthiophene), by Schimomura and co-workers [28, 25].

It is our aim to present graphical analysis of the linear and nonlinear dielectric relaxation of rigid, non-interacting symmetric-top dipolar molecules in spherical solvents, the phenomena which are strongly dependent both on the anisotropy of rotational diffusion tensor and on the angle between the molecular dipole and the symmetry axis. In Sect. 2 we derive the formula for the third-order electric polarization induced in the liquids by a strong external reorienting field, which consists of the sum of three terms connected with the "cubic" relaxation functions $A_{11}^{x x x}(t), A_{10}^{z z z}(t)$, and $A_{10}^{x x z}(t)+A_{11}^{x z z}(t)$. These functions depend on the parameter of the rotational diffusion anisotropy and on the shape of electric fields and are given for the nonlinear electric susceptibility in Langevin saturation, $\chi(-\omega ; \omega, 0,0)$, in Sect. 3. In Sect. 4 the linear dielectric relaxation is briefly summarized and in Sect. 5 we discuss our results for nonlinear relaxation.

Section 6 contains all the dispersion and absorption graphs. Our results indicate that the spherical-top approximation may be used only in special cases, especially in the nonlinear case, in which the symmetric shape of the molecule cannot be neglected.

\section{Third-order electric polarization induced in the dielectric medium}

We consider a dielectric system of the volume $V$ composed of a great number $N$ of dipolar molecules, with the permanent dipole moment of $\mu$. On neglecting the 
induced molecular polarizabilities and molecular interactions, the $Z$ component of the total dipole moment $M_{Z}\left[E_{Z}(t)\right]$ of the system, induced by the time-variable external electric field $E_{Z}(t)=E_{Z} g(t)$, is equal to

$$
\begin{aligned}
& M_{Z}\left[E_{Z}(t)\right] \\
& \quad=N \sqrt{\frac{2 \pi}{3}}\left[\mathrm{i} \mu_{x}\left(Y_{11}+Y_{1-1}\right)+\mu_{y}\left(Y_{11}-Y_{1-1}\right)-\sqrt{2} \mu_{z} Y_{10}\right] g(t),
\end{aligned}
$$

where $Y_{l m} \equiv Y_{l m}(\vartheta, \varphi)$ are the spherical harmonic functions, depending on the two angles between $E_{Z}(t)$ and the $z$ axis of the molecular coordinate set: the polar $\vartheta$ and the azimuthal $\varphi$ ones, $\mathrm{i}^{2}=-1$, and the function $g(t)$ describes the shape of the electric field. The components of the permanent dipole moment are: $\mu_{z}=\mu \cos \Theta, \mu_{x}=\mu \cos \Phi \sin \Theta, \mu_{y}=\mu \sin \Phi \sin \Theta$, where $\Theta, \Phi$ are the angles between $\mu$ and the $z$ axis of the molecular coordinate set.

The electric polarization $\left\langle P_{Z}(t)\right\rangle$ induced in the dielectric is given by the ensemble average

$$
\left\langle P_{Z}(t)\right\rangle=\frac{N}{4 \pi V} \int_{0}^{2 \pi} \int_{0}^{\pi} M_{Z}\left[E_{Z}(t)\right] f\left[\vartheta, \varphi ; E_{Z}(t)\right] \sin \vartheta \mathrm{d} \vartheta \mathrm{d} \varphi
$$

where $f\left[\vartheta, \varphi ; E_{Z}(t)\right]$ denotes the probability distribution function describing the rotational diffusion of a molecule.

We assume that the time evolution of the distribution function $f \equiv$ $f\left[\vartheta, \varphi ; E_{Z}(t)\right]$ is governed by the Smoluchowski-Debye rotational diffusion equation for the symmetric-top molecule $[19,20]$ :

$$
\begin{aligned}
& D_{z z}^{-1} \frac{\partial f}{\partial t}+\xi \hat{L}^{2} f+(1-\xi) \hat{L}_{z}^{2} f=\frac{1}{2 k T}\left[(\xi-1) \hat{L}_{z}^{2}(u f)-\xi \hat{L}^{2}(u f)\right. \\
& \left.\quad+(\xi-1) f \hat{L}_{z}^{2} u-\xi f \hat{L}^{2} u-(\xi-1) u \hat{L}_{z}^{2} f+\xi u \hat{L}^{2} f\right]
\end{aligned}
$$

where $\hat{L}$ and $\hat{L}_{Z}$ are the components of the quantum mechanical angular momentum operator but with $\hbar=1$ and $u \equiv u\left[\vartheta, \varphi ; E_{Z}(t)\right]$ denotes the change in the potential energy of the molecule in an external electric field

$$
u\left[\vartheta, \varphi ; E_{Z}(t)\right]=k T\left[\mathrm{i} p_{x}\left(Y_{11}+Y_{1-1}\right)+p_{y}\left(Y_{11}-Y_{1-1}\right)-p_{z} Y_{10}\right] g(t),
$$

where the dimensionless parameters of reorientation of the permanent dipole moment components are

$$
p_{\alpha}=\sqrt{\frac{2 \pi}{3}} \frac{\mu_{\alpha} E_{Z}}{k T}, \quad p_{z}=2 \sqrt{\frac{\pi}{3}} \frac{\mu_{z} E_{Z}}{k T},
$$

$k$ is the Boltzmann constant, $T$ - absolute temperature and $\alpha=x, y$. In the Smoluchowski Eq. (3) the dimensionless parameter of the rotational diffusion tensor anisotropy $\xi$ of symmetric-top molecule $-D_{x x}=D_{y y} \neq D_{z z}$ - is

$$
\xi=\frac{D_{x x}}{D_{z z}}
$$

where $D_{x x}, D_{z z}$ are the rotational diffusion constants around the molecular $x$ and 
$z$ axis, respectively. In the particular case of the spherical-top molecule $\xi=1$ and Eq. (3) becomes the well-known Smoluchowski-Debye equation depending on the polar angle $\vartheta$ only [9-11], which is fundamental for the theories of linear and nonlinear dielectric relaxation [6, 7], the dynamic Kerr effect [5], as well as many interesting magnetic relaxation phenomena [6].

The approximate solution of Eq. (3), if $p_{x}, p_{y}, p_{z} \ll 1$, known as "the low molecular reorientation", is given in $[10,22]$.

If the intensity of the external field $E_{Z}(t)$ is sufficiently high, it may induce in the dielectric, in addition to the linear polarization $\left\langle P_{Z}^{(1)}(t)\right\rangle$, proportional to $E_{Z}$ :

$$
\left\langle P_{Z}^{(1)}(t)\right\rangle=\left[A_{11}^{x}(t) \sin ^{2} \Theta+A_{10}^{z}(t) \cos ^{2} \Theta\right] \frac{\rho \mu^{2} E_{Z}}{3 k T},
$$

the third-order electric polarization, equal to $[9,10]$ :

$$
\begin{aligned}
& \left\langle P_{Z}^{(3)}(t)\right\rangle=-\left\{A_{11}^{x x x}(t) \sin ^{4} \Theta+A_{10}^{z z z}(t) \cos ^{4} \Theta\right. \\
& \left.+\left[A_{10}^{x x z}(t)+A_{11}^{x z z}(t)\right] \sin ^{2} \Theta \cos ^{2} \Theta\right\} \frac{\rho \mu^{4} E_{Z}^{3}}{45 k^{3} T^{3}} .
\end{aligned}
$$

For the "ideal" symmetric-top molecules, i.e. when $D_{x x}=D_{y y} \neq D_{z z}$ and $\mu_{x}=\mu_{y} \neq \mu_{z}$, too, or simply $\Theta=\pi / 4$, Eqs. (7), (8) are reduced to

$$
\left\langle P_{Z}^{(1)}(t)\right\rangle=\left[A_{11}^{x}(t)+A_{10}^{z}(t)\right] \frac{\rho \mu^{2} E_{Z}}{6 k T}
$$

and

$$
\left\langle P_{Z}^{(3)}(t)\right\rangle=-\left[A_{11}^{x x x}(t)+A_{10}^{x x z}(t)+A_{11}^{x z z}(t)+A_{10}^{z z z}(t)\right] \frac{\rho \mu^{4} E_{Z}^{3}}{180 k^{3} T^{3}} .
$$

The linear $A_{11}^{x}(t), A_{10}^{z}(t)$ and "cubic" $A_{11}^{x x x}(t), A_{10}^{z z z}(t), A_{10}^{x x z}(t), A_{11}^{x z z}(t)$ relaxational functions, depending on the shape of the external reorienting electric fields $g(t)$, parameter $\xi$ of the rotational tensor anisotropy and the rotational relaxation times of the symmetric-top molecule $\tau_{l m}$, can be found from the set of simple linear differential equations [9, 22], resulting from the Smoluchowski-Debye Eq. (3).

Therefore linear relaxation consists, Eq. (7), of two terms. It is seen from Eq. (8) that nonlinear Langevin relaxation is a superposition of three independent terms. Our treatment may be extended to the case of the polarizable molecules, taking into account the additional term in the potential energy $u\left[\vartheta, \varphi ; E_{Z}(t)\right]$ in Eq. (4).

\section{The rotational diffusion functions of symmetric-top molecule in the presence of constant and harmonic electric fields}

Let us consider an external reorienting electric field $E_{Z}(t)$ as a sum of constant and harmonic fields

$$
g(t)=1+\frac{1}{2} \lambda\left(\mathrm{e}^{-\mathrm{i} \omega t}+\mathrm{e}^{\mathrm{i} \omega t}\right), \quad \lambda=\frac{E_{\omega}}{E_{Z}}
$$

switching on at the time $t=0$. Here $\omega$ denotes the frequency of the electric field 
$E_{\omega} \cos \omega t$. The extension of the Kielich method [9-11, 22] to the symmetric-top molecules gives the steady-state result

$$
2 A_{11}^{x}(t)=1+\lambda R_{11}^{\omega} \mathrm{e}^{-\mathrm{i} \omega t}+\text { c.c. }, \quad 2 A_{10}^{z}(t)=1+\lambda R_{10}^{\omega} \mathrm{e}^{-\mathrm{i} \omega t}+\text { c.c. }
$$

in which the complex Debye-Kielich factors $R_{l m}(n \omega) \equiv R_{l m}^{n \omega}$, describing the dispersion and absorption of the medium are defined as

$$
R_{l m}^{n \omega}=\left(1-\mathrm{i} n \omega \tau_{l m}\right)^{-1} \text {. }
$$

Here the rotational relaxation times of the symmetric-top molecule $\tau_{l m}$ are equal to

$$
\tau_{l m}=\left[l(l+1) D_{z z}-m^{2}\left(D_{z z}-D_{x x}\right)\right]^{-1}
$$

and the abbreviation "c.c." denotes the complex conjugate term. In this paper we neglect transient effects in the rise in time of polarization and describe here only the steady-state polarization, attained by the dielectric medium after a sufficiently long time $t \gg \tau_{10}$. In Fig. 1 we plot the ratios

$$
\frac{\tau_{11}}{\tau_{10}}=\frac{2}{1+\xi}, \quad \frac{\tau_{21}}{\tau_{20}}=\frac{6}{5+\xi},
$$

and

$$
\frac{\tau_{22}}{\tau_{20}}=\frac{3}{1+2 \xi}
$$

versus the parameter of anisotropy of the rotational diffusion components $\xi$. We see that for $\xi<1$ all these ratios are greater than 1 and for $\xi \rightarrow \infty$ these ratios decrease to zero.

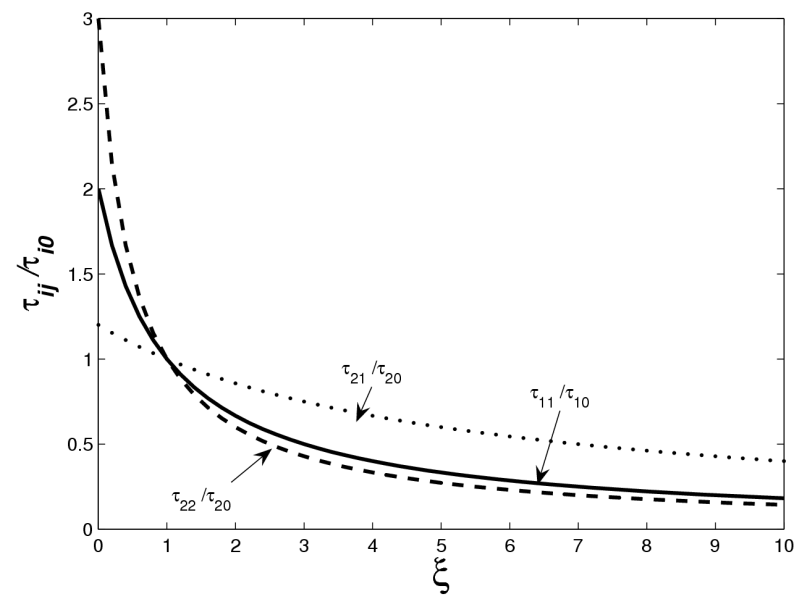

Fig. 1. Relaxation times versus parameters anisotropy $\xi$.

The cubic reorientational functions $A_{l m}^{\alpha \alpha \alpha}(t)$ for the symmetric-top molecules are of more complicated form and may be obtained from the set of linear differential equations, given in [22]. We have this result 


$$
\begin{aligned}
& 2 A_{10}^{z z z}(t)=1+\lambda R_{10}^{\omega}\left(1+R_{20}^{\omega}+R_{10}^{\omega} R_{20}^{\omega}\right) \mathrm{e}^{-\mathrm{i} \omega t} \\
& +\frac{1}{2} \lambda^{2}\left(R_{10}^{\omega}+R_{20}^{\omega}+R_{10}^{\omega} R_{20}^{\omega}\right) \\
& +\frac{1}{2} \lambda^{2}\left(R_{10}^{\omega} R_{10}^{2 \omega} R_{20}^{2 \omega}+R_{10}^{\omega} R_{10}^{2 \omega} R_{20}^{\omega}+R_{10}^{2 \omega} R_{20}^{\omega}\right) \mathrm{e}^{-\mathrm{i} 2 \omega t} \\
& +\frac{1}{4} \lambda^{3} R_{10}^{\omega}\left(R_{10}^{\omega}+R_{10}^{-\omega}+R_{20}^{2 \omega}\right) \mathrm{e}^{-\mathrm{i} \omega t} \\
& +\frac{1}{4} \lambda^{3} R_{10}^{\omega} R_{10}^{3 \omega} R_{20}^{2 \omega} \mathrm{e}^{-\mathrm{i} 3 \omega t}+\text { c.c. }
\end{aligned}
$$

The function $A_{10}^{z z z}(t)$ is the one that determines the rotational relaxation of spherical-top molecules with $\mu_{z} \neq 0, \mu_{x}=\mu_{y}=0$, too, and depends on two relaxation times

$$
\tau_{10}=\left(2 D_{z z}\right)^{-1}, \quad \tau_{20}=\left(6 D_{z z}\right)^{-1} .
$$

Moreover we obtain

$$
\begin{aligned}
& 4(1+\xi)\left[A_{11}^{x x x}(t)-1\right]=\lambda R_{11}^{\omega}\left\{1+\xi+\left(1+R_{11}^{\omega}\right)\left[3 R_{22}^{\omega}+(2 \xi-1) R_{20}^{\omega}\right]\right\} \mathrm{e}^{-\mathrm{i} \omega t} \\
& \quad+\frac{1}{2} \lambda^{2}\left\{2(1+\xi) R_{11}^{\omega} R_{11}^{\omega}+\left(1+R_{11}^{\omega}\right)\left[3 R_{22}^{\omega}+(2 \xi-1) R_{20}^{\omega}\right]\right\} \\
& \quad+\frac{1}{2} \lambda^{2}\left\{3 R_{11}^{\omega} R_{22}^{2 \omega}+(2 \xi-1) R_{20}^{2 \omega}+\left(1+R_{11}^{\omega}\right) R_{11}^{2 \omega}\left[3 R_{22}^{\omega}+(2 \xi-1) R_{20}^{\omega}\right]\right\} \mathrm{e}^{-\mathrm{i} 2 \omega t} \\
& \quad+\frac{1}{4} \lambda^{3} R_{11}^{\omega}\left[3 R_{11}^{\omega} R_{22}^{2 \omega}+2(1-\xi)\left(2+R_{20}^{2 \omega}\right)\right] \mathrm{e}^{-\mathrm{i} \omega t} \\
& \quad+\frac{1}{4} \lambda^{3} R_{11}^{3 \omega}\left[3 R_{11}^{\omega} R_{22}^{2 \omega}+2(\xi-1) R_{20}^{2 \omega}\right] \mathrm{e}^{-\mathrm{i} 3 \omega t}+\text { c.c. }
\end{aligned}
$$

and

$$
\begin{aligned}
& 2 A_{10}^{x x z}(t)=1+\frac{1}{4} \lambda\left[2-\left(1+R_{11}^{\omega}\right) R_{20}^{\omega}+3\left(1+a R_{10}^{\omega}+b R_{11}^{\omega}\right) R_{21}^{\omega}\right] \mathrm{e}^{-\mathrm{i} \omega t} \\
& \quad+\frac{1}{8} \lambda^{2}\left[3\left(a R_{10}^{\omega}+b R_{11}^{\omega}\right)\left(1+R_{21}^{\omega}\right)+3 R_{21}^{\omega}-R_{11}^{\omega}-\left(1+R_{11}^{\omega}\right) R_{20}^{\omega}\right] \\
& \quad+\frac{1}{8} \lambda^{2}\left[6\left(1+a R_{10}^{\omega}+b R_{21}^{\omega}-2 R_{11}^{\omega} R_{20}^{2 \omega}-R_{20}^{\omega}\right) R_{10}^{2 \omega} \mathrm{e}^{-\mathrm{i} 2 \omega t}\right] \\
& \quad+\frac{1}{16} \lambda^{3}\left\{R_{10}^{\omega}\left[3\left(a R_{10}^{\omega}+b R_{11}^{\omega}\right)\left(1+R_{21}^{\omega}\right)-R_{11}^{\omega}\left(1+R_{20}^{2 \omega}\right)\right]\right. \\
& \left.\quad+R_{10}^{-\omega}\left[3\left(a R_{10}^{-\omega}+b R_{11}^{-\omega}\right)-R_{11}^{-\omega}\right]\right\} \mathrm{e}^{-\mathrm{i} \omega t} \\
& \quad+\frac{1}{16} \lambda^{3}\left[3\left(a R_{10}^{\omega}+b R_{11}^{\omega}\right) R_{21}^{2 \omega}-R_{11}^{\omega} R_{20}^{2 \omega}\right] R_{10}^{3 \omega} \mathrm{e}^{-\mathrm{i} 3 \omega t}+\text { c.c. },
\end{aligned}
$$

where the coefficients $a, b$ are equal to

and finally

$$
a=\frac{1+2 \xi}{1+5 \xi}, \quad b=\frac{3 \xi}{1+5 \xi}
$$




$$
\begin{aligned}
(1 & +\xi)\left[A_{11}^{x z z}(t)-1\right]=\frac{1}{2} \lambda\left[\left(1+R_{11}^{\omega}\right) R_{20}^{\omega}-2 \xi\left(1+R_{10}^{\omega}\right) R_{20}^{\omega}\right. \\
& \left.+(1+\xi) R_{11}^{-\omega}+3 \xi\left(1+a R_{10}^{\omega}+b R_{11}^{\omega}\right) R_{21}^{\omega}\right] \mathrm{e}^{-\mathrm{i} \omega t} \\
& +\frac{1}{4} \lambda^{2}\left[R_{11}^{\omega}-2 \xi R_{10}^{\omega}+3 \xi\left(a R_{10}+b R_{11}^{\omega}\right)+\left(1+R_{11}^{\omega}\right) R_{20}^{\omega}\right. \\
& \left.-2 \xi\left(1+R_{10}^{\omega}\right) R_{20}^{\omega}+3 \xi\left(1+a R_{10}^{\omega}+b R_{11}^{\omega} R_{21}^{\omega}\right)\right] \\
& +\frac{1}{4} \lambda^{2}\left[\left(1+R_{11}^{\omega}\right) R_{20}^{\omega}-2 \xi R_{20}^{\omega}\left(1+R_{10}^{\omega} R_{20}^{\omega}\right)+3 \xi\left(1+a R_{10}^{\omega}+b R_{11}^{\omega}\right) R_{21}^{\omega}\right. \\
& \left.+R_{11}^{\omega} R_{20}^{2 \omega}-2 \xi R_{10}^{\omega} R_{20}^{2 \omega}+3 \xi\left(a R_{10}^{\omega}+b R_{11}^{\omega}\right) R_{21}^{2 \omega}\right] R_{21}^{2 \omega} \mathrm{e}^{-\mathrm{i} 2 \omega t} \\
& +\frac{1}{8} \lambda^{3}\left[R_{11}^{\omega}+R_{11}^{-\omega}-2 \xi\left(R_{10}^{\omega}+R_{10}^{-\omega}\right)+3 \xi a\left(R_{10}^{\omega}+R_{10}^{-\omega}\right)+3 \xi b\left(R_{11}^{\omega}+R_{11}^{-\omega}\right)\right. \\
& \left.+R_{11}^{\omega} R_{20}^{2 \omega}-2 \xi R_{10}^{\omega} R_{20}^{2 \omega}+3 \xi\left(a R_{10}+b R_{11}^{\omega}\right) R_{21}^{2 \omega}\right] R_{11}^{\omega} \mathrm{e}^{-\mathrm{i} \omega t} \\
& +\frac{1}{8} \lambda^{3}\left[R_{11}^{\omega} R_{20}^{2 \omega}-2 \xi R_{10}^{\omega} R_{20}^{2 \omega}+3 \xi\left(a R_{10}^{\omega}+b R_{11}^{\omega}\right) R_{21}^{2 \omega}\right] R_{11}^{3 \omega} \mathrm{e}^{-\mathrm{i} 3 \omega t}+\text { c.c. } \quad(20)
\end{aligned}
$$

Equations (15)-(20) represent the essential result of our theory and describe the steady-state of the third-order electric polarization induced in the dielectric medium by a sum of the constant and the harmonic fields, Eq. (11). The dispersion and absorption depend on the symmetric-top molecule orientational relaxation times $\tau_{10}, \tau_{11}, \tau_{20}, \tau_{21}, \tau_{22}$ as well as on the complex, nonlinear Debye-Kielich factors $R_{l m}^{n \omega}$ given by Eq. (13). It is seen from Eqs. (7),(8),(15)-(20) that the anisotropy of the rotational diffusion components $\xi$ may have great significance in the theory of nonlinear dielectric relaxation.

The time independent component of the third-ordered electric polarization Eq. (8) is connected with the term proportional to $\lambda^{0}$, and may be obtained, in the case of spherical molecules with $\mu_{x}=\mu_{y}=0, \mu_{z} \neq 0$, from the series expansion of the well-known Langevin function $L(y)$, where

$$
\begin{aligned}
& \left\langle P_{Z}\left(E_{Z}\right)\right\rangle=\rho\left(\operatorname{coth} y-\frac{1}{y}\right)=\rho L(y) \cong \rho\left(\frac{y}{3}-\frac{y^{3}}{45}+\ldots\right), \\
& y=\frac{\mu_{z} E_{Z}}{k T} .
\end{aligned}
$$

We see that the terms in Eqs. (12),(15)-(20) proportional to $\lambda$ change with the fundamental frequency $\omega$. These components of polarization (8) are measured in the NDE [14-16] firstly observed in 1936 as "the static positive NDE" in nitrobenzene solutions by Piekara [12]. These studies have soon a long history and are quoted in some monographic papers [7, 8, 13].

The terms proportional to $\lambda^{2}$ in Eqs. (12), (15)-(20) split into two components - the first, time-independent, and the second, changing with the double frequency $2 \omega$, which describes the generation of the second harmonic polarization 
in the presence of a strong dc electric field. Various third-order electric polarization phenomena for the spherical-top molecules were discussed in [9-11].

\section{Linear dielectric relaxation of non-interacting, rigid, dipolar and symmetric-top molecules}

The external electric field Eq. (11) induces, according to Eq. (9) the first-order polarization $\left\langle P^{(1)}(E)\right\rangle$, which may be written as a sum of two components

$$
\left\langle P_{Z}^{(1)}[E(t)]\right\rangle=\left\langle P_{Z}^{(1)}\left(E_{Z}\right)\right\rangle+\left\langle P_{Z}^{(1)}\left[E_{\omega}(t)\right]\right\rangle,
$$

the time-independent polarization

$$
\left\langle P_{Z}^{(1)}\left(E_{Z}\right)\right\rangle=\frac{\rho \mu^{2} E_{Z}}{3 k T}
$$

and a harmonic polarization, changing with the fundamental frequency $\omega$, which, according to Eqs. (7),(12) may be written in the form

$$
\begin{aligned}
& \left\langle P_{Z}^{(1)}\left[E_{\omega}(t)\right]\right\rangle \\
& \quad=\left[r_{11}^{\omega} \cos \left(\omega t-\psi_{11}^{\omega}\right) \sin ^{2} \Theta+r_{10}^{\omega} \cos \left(\omega t-\psi_{10}^{\omega}\right) \cos ^{2} \Theta\right] \frac{\rho \mu^{2} E_{\omega}}{3 k T} .
\end{aligned}
$$

This harmonic term depends on the real dispersional functions

$$
r_{l m}(n \omega) \equiv r_{l m}^{n \omega}=\left[1+\left(n \omega \tau_{l m}\right)^{2}\right]^{-\frac{1}{2}}
$$

and the phase shift angles

$$
\begin{aligned}
& \cos \psi_{l m}^{n \omega}=r_{l m}^{n \omega}, \\
& \sin \psi_{l m}^{n \omega}=n \omega \tau_{l m} r_{l m}^{n \omega}
\end{aligned}
$$

as well as on the relaxation times $\tau_{10}, \tau_{11}$.

It is worth noting that Eq. (22) is a special case of the old Perrin formula [2] for linear electric polarization of the dielectric medium consisting of noninteracting rigid, dipolar and asymmetric-top molecules in a cosine electric field

$$
\left\langle P_{Z}^{(1)}(t)\right\rangle=\frac{\rho E_{\omega}}{3 k T} \sum_{\alpha=x, y, z} \frac{\mu_{\alpha}^{2} \mathrm{e}^{\mathrm{i} \omega t}}{1+\mathrm{i} \omega \tau_{\alpha}} .
$$

Here $\tau_{\alpha}$ are the rotational relaxation times of the asymmetric-top molecule around its $\alpha$-axis, respectively. This result was extended for the interesting case of asymmetric-top molecules with the rotating dipole groups by Budo and co-workers [3].

The harmonic component (22) of the linear polarization may be written as

$$
\left\langle P_{Z}^{(1)}\left(E_{\omega}\right)\right\rangle=\epsilon_{0} \chi^{*}(-\omega ; \omega) E_{\omega} g(t),
$$

where $\chi^{*}(-\omega ; \omega)$ is the complex linear electric susceptibility component with frequency $\omega$ of the medium, $\epsilon_{0}$ is the dielectric permittivity of the vacuum. The complex linear susceptibility $\chi^{*}(-\omega ; \omega)$ is a sum of the real $\chi^{\prime}(-\omega ; \omega)$ and the imaginary $\chi^{\prime \prime}(-\omega ; \omega)$ parts

$$
\chi^{*}(-\omega ; \omega)=\chi^{\prime}(-\omega ; \omega)-\mathrm{i} \chi^{\prime \prime}(-\omega ; \omega),
$$

which may be written, according to Eqs. (22)-(24) in the form 


$$
3 \epsilon_{0} k T \chi^{\prime}(-\omega ; \omega)=\rho \mu^{2}\left(s_{11}^{\omega} \sin ^{2} \Theta+s_{10}^{\omega} \cos ^{2} \Theta\right)
$$

and

$$
3 \epsilon_{0} k T \chi^{\prime \prime}(-\omega ; \omega)=-\rho \mu^{2}\left(\omega \tau_{11} s_{11}^{\omega} \sin ^{2} \Theta+\omega \tau_{10} s_{10}^{\omega} \cos ^{2} \Theta\right),
$$

where $s_{l m}^{n \omega}$ are the quadratic Debye-Kielich dispersional functions

$$
s_{l m}^{n \omega} \equiv s_{l m}(n \omega)=\left[r_{l m}^{n \omega}\right]^{2}=\left(1+n^{2} \omega^{2} \tau_{l m}^{2}\right)^{-1},
$$

which decrease from the maximal value $s_{l m}^{n \omega}(0)=1$ for $\omega=0$ to zero when $\omega \tau_{l m} \rightarrow \infty$.

Equations (31), (32) describe the linear dielectric relaxation as a superposition of two rotational motions around two perpendicular molecular axes of symmetry. Those rotational motions were observed, for example, by Jadżyn and co-workers [17], in linear dielectric spectroscopy with the solutions of 4$n$-decyloxyphenyl-4'-cyano-benzoate (DOPCB) (or $\mathrm{C}_{10} \mathrm{H}_{21}$-O-Ph-OOC-PH-CN) molecules, for which the dispersional and absorptional spectra are the sum of two components, corresponding to the rotational diffusion around the short and long symmetry axes.

\section{Nonlinear dielectric relaxation in dilute solution of dipolar, symmetric-top molecules in spherical solvent}

In this section we will discuss the properties of dispersion and absorption of the Langevin saturation measured in NDE in a dilute solution of dipolar, symmetric-top molecules in spherical solvents. These accurate methods of modern nonlinear dielectric spectroscopy permit measurements of such small effects $[14-16]$.

The NDE consists on the induction in the dielectric medium of the nonlinear polarization by the strong constant field $E_{Z}$ and a weak measuring harmonic field $E_{\omega} \cos \omega t$, Eq. (11), with $\lambda \ll 1$. In this case we can neglect in the relaxational functions (15)-(20) all the terms proportional to $\lambda^{2}$ and $\lambda^{3}$, and the result is

$$
\begin{aligned}
& A_{10}^{z z z}(t)=1+\lambda r_{10}\left[\cos \left(\omega t-\psi_{10}\right)\right. \\
& \left.\quad+r_{20} \cos \left(\omega t-\psi_{10}-\psi_{20}\right)+r_{10} r_{20} \cos \left(\omega t-2 \psi_{10}-\psi_{20}\right)\right] \\
& 4(1+\xi)\left[A_{11}^{x x x}(t)-1\right]=\lambda r_{11}\left[(1+\xi) \cos \left(\omega t-\psi_{11}\right)\right. \\
& \quad+3 r_{22} \cos \left(\omega t-\psi_{11}-\psi_{22}\right)+(2 \xi-1) r_{20} \cos \left(\omega t-\psi_{11}-\psi_{20}\right) \\
& \left.\quad+3 r_{11} r_{22} \cos \left(\omega t-2 \psi_{11}-\psi_{22}\right)+(2 \xi-1) r_{11} r_{20} \cos \left(\omega t-2 \psi_{11}-\psi_{20}\right)\right],(35) \\
& 2 A_{10}^{x x z}(t)-1=\frac{1}{4} \lambda\left[2 \cos \omega t-r_{20} \cos \left(\omega t-\psi_{20}\right)-r_{11} r_{20} \cos \left(\omega t-\psi_{11}-\psi_{20}\right)\right. \\
& \quad+3 r_{21} \cos \left(\omega t-\psi_{21}\right)+3 a r_{10} r_{21} \cos \left(\omega t-\psi_{21}\right) \\
& \left.\quad+3 b r_{11} r_{21} \cos \left(\omega t-\psi_{11}-\psi_{21}\right)\right]
\end{aligned}
$$




$$
\begin{aligned}
& 2(1+\xi)\left[A_{11}^{x z z}(t)-1\right]=\lambda\left[(1-2 \xi) r_{20} \cos \left(\omega t-\psi_{20}\right)\right. \\
& \quad+r_{11} r_{20} \cos \left(\omega t-\psi_{11}-\psi_{20}\right)-2 \xi r_{10} r_{20} \cos \left(\omega t-\psi_{10}-\psi_{20}\right) \\
& \quad+(1+\xi) r_{11} \cos \left(\omega t-\psi_{11}\right)+3 \xi r_{21} \cos \left(\omega t-\psi_{21}\right) \\
& \left.\quad+3 a \xi r_{10} r_{21} \cos \left(\omega t-\psi_{10}-\psi_{21}\right)+3 b \xi r_{11} r_{21} \cos \left(\omega t-\psi_{11}-\psi_{21}\right)\right] .
\end{aligned}
$$

It is seen from Eqs. (34)-(37) that only $A_{10}^{z z z}(t)$ does not depend on the anisotropy parameter $\xi$.

The third-order electric polarization (8) may be written in the form

$$
\left\langle P_{Z}^{(3)}\left(E_{\omega}, E_{Z}^{2}\right)\right\rangle=\left[\chi^{\prime}(-\omega ; \omega, 0,0) \cos \omega t-\chi^{\prime \prime}(-\omega ; \omega, 0,0) \sin \omega t\right] E_{\omega} E_{Z}^{2},
$$

where the real $\chi^{\prime}(-\omega ; \omega, 0,0)$ and imaginary $\chi^{\prime \prime}(-\omega ; \omega, 0,0)$ parts of the complex nonlinear electric susceptibility are equal to

$$
\begin{gathered}
\chi^{\prime}(-\omega ; \omega, 0,0)=-\left\{a_{11}^{x x x}(\omega, \xi) \sin ^{4} \Theta+a_{10}^{z z z}(\omega) \cos ^{4} \Theta\right. \\
\left.+\left[a_{10}^{x x z}(\omega, \xi)+a_{11}^{x z z}(\omega, \xi)\right] \sin ^{2} \Theta \cos ^{2} \Theta\right\} \frac{\rho \mu^{4}}{45 k^{3} T^{3}}
\end{gathered}
$$

and

$$
\begin{gathered}
\chi^{\prime \prime}(-\omega ; \omega, 0,0)=\left\{b_{11}^{x x x}(\omega, \xi) \sin ^{4} \Theta+b_{10}^{z z z}(\omega) \cos ^{4} \Theta\right. \\
\left.\left.+\left[b_{10}^{x x z}(\omega, \xi)+b_{11}^{x z z} \omega, \xi\right)\right] \sin ^{2} \Theta \cos ^{2} \Theta\right\} \frac{\rho \mu^{4}}{45 k^{3} T^{3}} .
\end{gathered}
$$

The dispersional behavior and the influence of the molecular shape on the nonlinear susceptibility are described by the functions

$$
\begin{aligned}
& 4(1+\xi) a_{11}^{x x x}(\omega, \xi)=s_{11}\left\{1+\xi+3 s_{22}\left[2 s_{11}-\omega^{2} \tau_{11} \tau_{22}\left(1+2 s_{11}\right)\right]\right. \\
& \left.\quad+(2 \xi-1) s_{20}\left[2 s_{11}-\omega^{2} \tau_{11} \tau_{20}\left(1+2 s_{11}\right)\right]\right\} \\
& a_{10}^{z z z}(\omega)=s_{10}\left\{1+s_{20}\left[2 s_{10}-\omega^{2} \tau_{10} \tau_{20}\left(1+2 s_{10}\right)\right]\right\} \\
& 8(1+\xi)\left[a_{10}^{x x z}(\omega, \xi)+a_{11}^{x z}(\omega, \xi)\right] \\
& \quad=2(1+\xi)-4(1+\xi) s_{11}+3(\xi-1) s_{20}+3(1+5 \xi) s_{21} \\
& \quad+(3-\xi) s_{11} s_{20}\left(1-\omega^{2} \tau_{11} \tau_{20}\right)+3(1+2 \xi) s_{10} s_{21}\left(1-\omega^{2} \tau_{10} \tau_{21}\right) \\
& \quad+9 \xi s_{11} s_{21}\left(1-\omega^{2} \tau_{11} \tau_{21}\right)+2 \xi s_{10} s_{20}\left(1-\omega^{2} \tau_{10} \tau_{20}\right), \\
& 4(1+\xi) b_{11}^{x x x}(\omega, \xi)=\omega s_{11}\left\{(1+\xi) \tau_{11}\right\}+3 s_{22}\left[\tau_{11}+2 s_{11}\left(\tau_{11}+\tau_{22}\right)\right] \\
& \left.\quad+(2 \xi-1) s_{20}\left[\tau_{11}+2 s_{11}\left(\tau_{11}+\tau_{20}\right)\right]\right\}, \\
& b_{10}^{z z z}(\omega)=\omega s_{10}\left\{\tau_{10}+s_{20}\left[\tau_{10}+2 s_{10}\left(\tau_{10}+\tau_{20}\right)\right]\right\}, \\
& 8(1+\xi)\left[b_{10}^{x x z}(\omega, \xi)+b_{11}^{x z z}(\omega, \xi)\right] \\
& \quad=\omega\left\{3(\xi-1) \tau_{20} s_{20}-4(1+\xi) \tau_{11} s_{11}\right.
\end{aligned}
$$




$$
\begin{aligned}
& +3(1+5 \xi) \tau_{21} s_{21}+(3-\xi)\left(\tau_{11}+\tau_{20}\right) s_{11} s_{20} \\
& +3(1+2 \xi)\left(\tau_{10}+\tau_{21}\right) s_{10} s_{21}+9 \xi\left(\tau_{11}+\tau_{21}\right) s_{11} s_{21} \\
& \left.+2 \xi\left(\tau_{10}+\tau_{20}\right) s_{10} s_{20}\right\} .
\end{aligned}
$$

In Eqs. (41)-(46) we ommitted the upper index $n$ in $s_{l m}^{n \omega}$, which is 1. Equations (39)-(46) are the main result of this paper and will be used for detailed graphical analysis of the influence of the symmetric-top shape of the molecule and of the orientation angle $\Theta$ between its permanent dipole and the $z$-molecular axis, on the linear and nonlinear dielectric dispersion and absorption in liquids.

We see from Eqs. (31),(32), (39), (40) that in the special case, when $\Theta=0$, the dielectric relaxation processes do not depend on the anisotropy parameter $\xi$, so the spherical-top Smoluchowski-Debye equation is a very good aproximation for the molecules with $\Theta=0$ but with $\xi$ different from 1 .

Our Eqs. (39), (40) are equivalent to the results of Kalmykov [23], Eq. (32). In his paper Kalmykov shows that the Euler-Langevin equation of the rotational Brownian motion for an asymmetric-top molecule may be reduced to the Smoluchowski-Debye equation. With his elegant method, the solution obtained with the use of the Wigner $D_{m n}^{j}(\alpha, \beta, \gamma)$ functions, the experimental investigations [16], performed by Jadżyn and co-workers, of the dielectric increments of dilute solutions of mesogenic 10-TPEB molecules, for which $\Theta=42^{\circ} \pm 2^{\circ}$, were successfully explained and the value of the rotational diffusion anisotropy parameter was evaluated as $\xi=8.7$ for this molecule.

\section{Graphical analysis of the linear and nonlinear dielectric relaxation processes}

Our aim is to analyze the changes in the Debye - linear and Langevin nonlinear dielectric relaxations, involved by symmetric shape of the molecules, in comparison with the relaxations of spherical molecules. All plotted linear susceptibilities, are normalized according to Eqs. (31), (32) with

$$
\frac{\rho \mu^{2}}{3 \varepsilon_{0} k T}=1 .
$$

Similarly for the nonlinear susceptibilities, Eqs. (39),(40), the normalization is

$$
\frac{\rho \mu^{4}}{45 k^{3} T^{3}}=1 .
$$

Figure 2 presents the dependences of the susceptibility $\chi^{\prime}(-\omega ; \omega)$, Eq. (31), on the frequency $\omega \tau_{20}$ of the ac electric field, in logarithmic scale, and the polar angle $\Theta$ between permanent dipole moment and the symmetry axis of the molecule, for some values of the anisotropy parameter $\xi$. For $\Theta=0$ all dispersion curves are, for all values of $\xi$, identical - it is simply the case of the SmoluchowskiDebye equation for the spherical top. The influence of the symmetric shape of the 


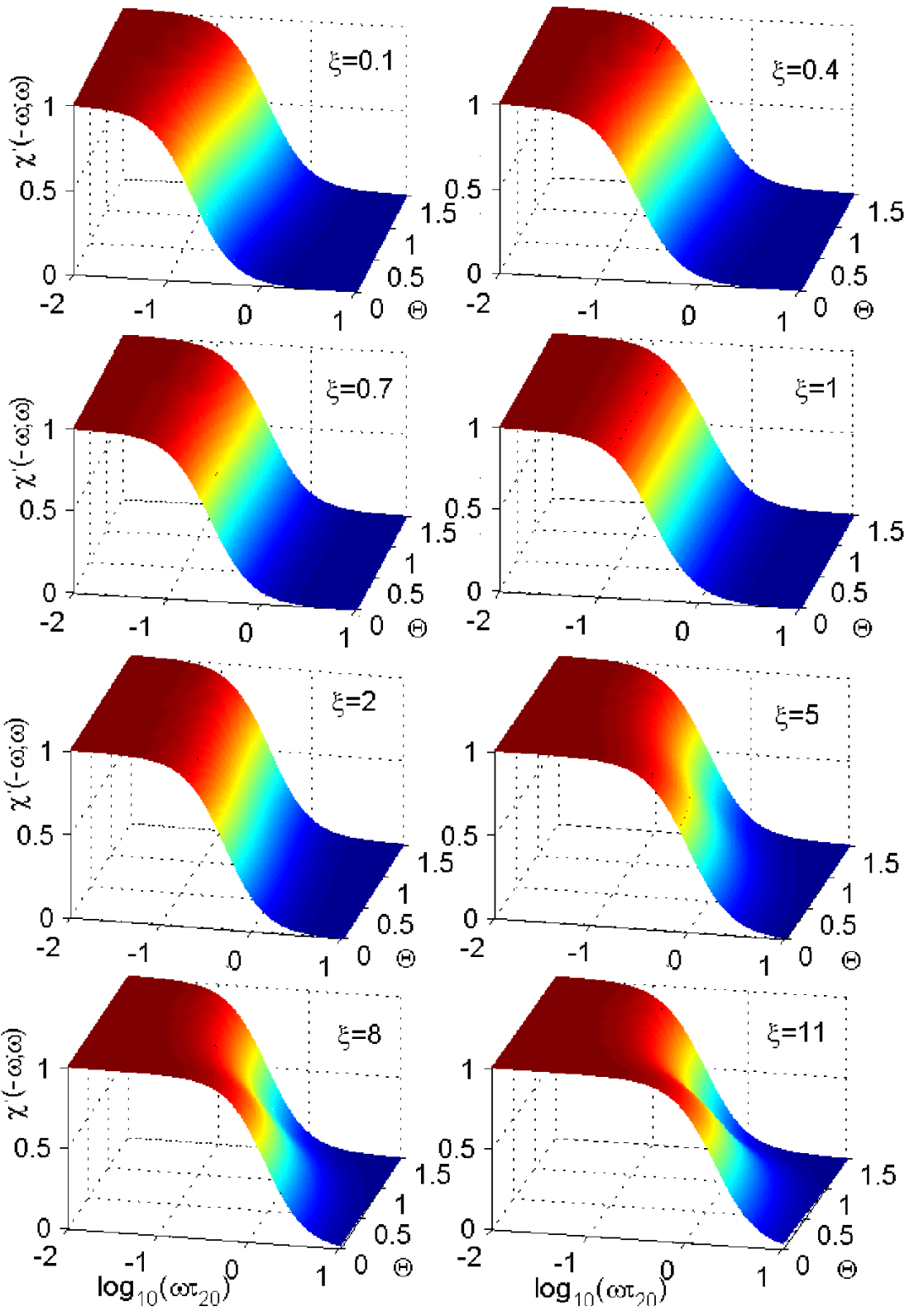

Fig. 2. Dependence of the linear susceptibility $\chi^{\prime}(-\omega ; \omega)$, given by Eq. (31), of the frequency $\omega \tau_{20}$ of the ac electric field and of the angle $\Theta$ between permanent dipole moment and the symmetry axis of the molecule - for some values of the anisotropy parameter $\xi$. The angle $\Theta$ is changing from 0 to $\pi / 2$.

molecule on $\chi^{\prime}(-\omega ; \omega)$ is stronger for $\xi>1$, for $\xi<1$ the changes are smaller. With increasing $\Theta$, the region of dispersion shifts to lower frequencies, as we can 


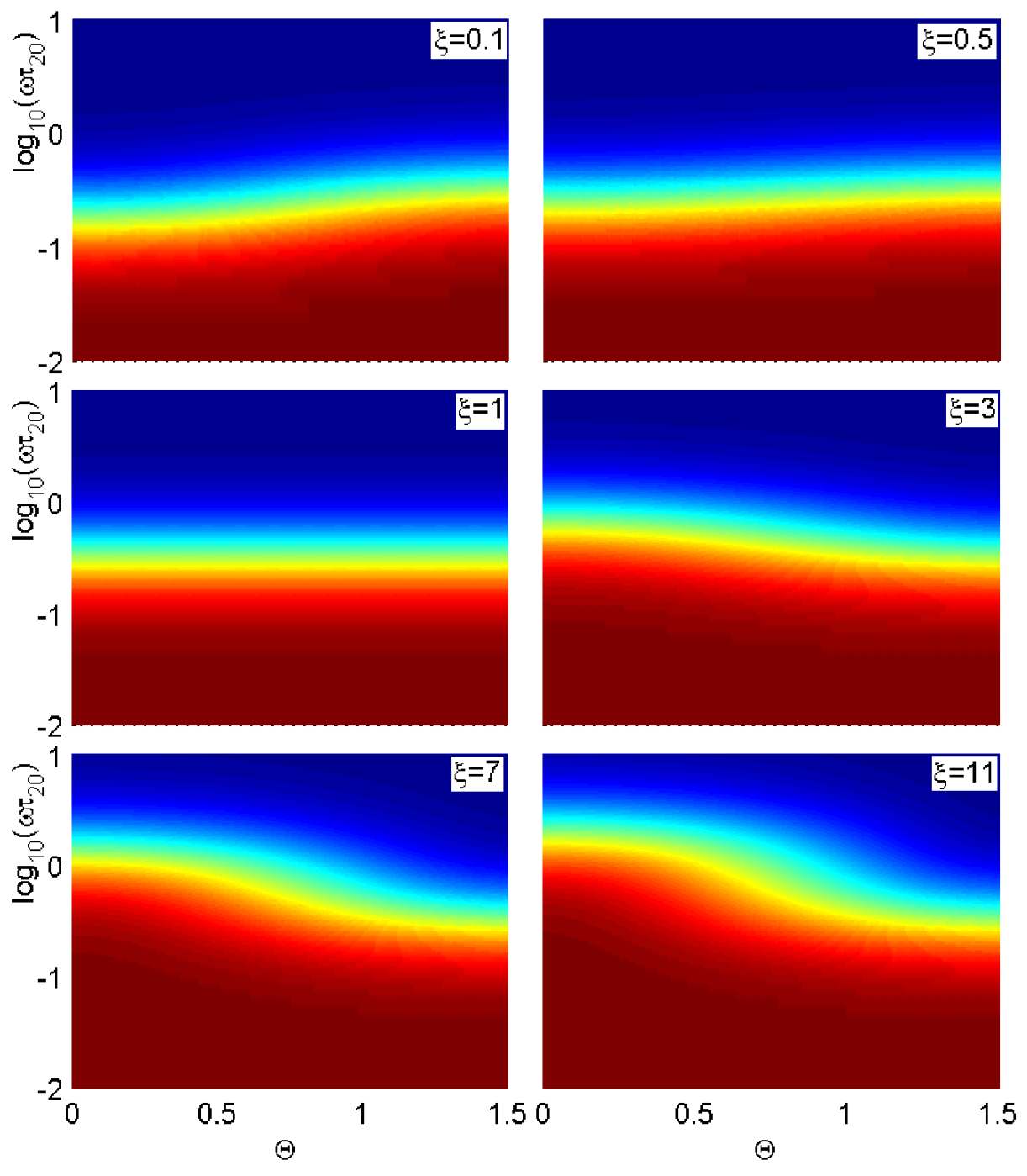

Fig. 3. Maps - projection of the linear susceptibility $\chi^{\prime}(-\omega ; \omega)$, given by Eq. (31) on the plane $\left(\Theta, \log _{10}\left(\omega \tau_{20}\right)\right)$.

see from Fig. 3, showing the projection of the linear susceptibility $\chi^{\prime}(-\omega ; \omega)$, given by Eq. (31), onto the plane $\left(\Theta, \log _{10}\left(\omega \tau_{20}\right)\right)$. Appriopriate colors or shades on all figures in our paper are connected with the values of susceptibilities.

Figure 4 presents the dependences of the linear susceptibility $\chi^{\prime \prime}(-\omega ; \omega)$, given by Eq. (32), of the frequency $\omega \tau_{20}$ of the ac electric field and of the angle $\Theta$ - for some values of the anisotropy parameter $\xi$, and similarly Fig. (5) gives the projection of the linear susceptibility $\chi^{\prime \prime}(-\omega ; \omega)$, given by Eq. (32) onto the plane $\left(\Theta, \log _{10}\left(\omega \tau_{20}\right)\right)$. These linear absorption curves are strongly changed, in 

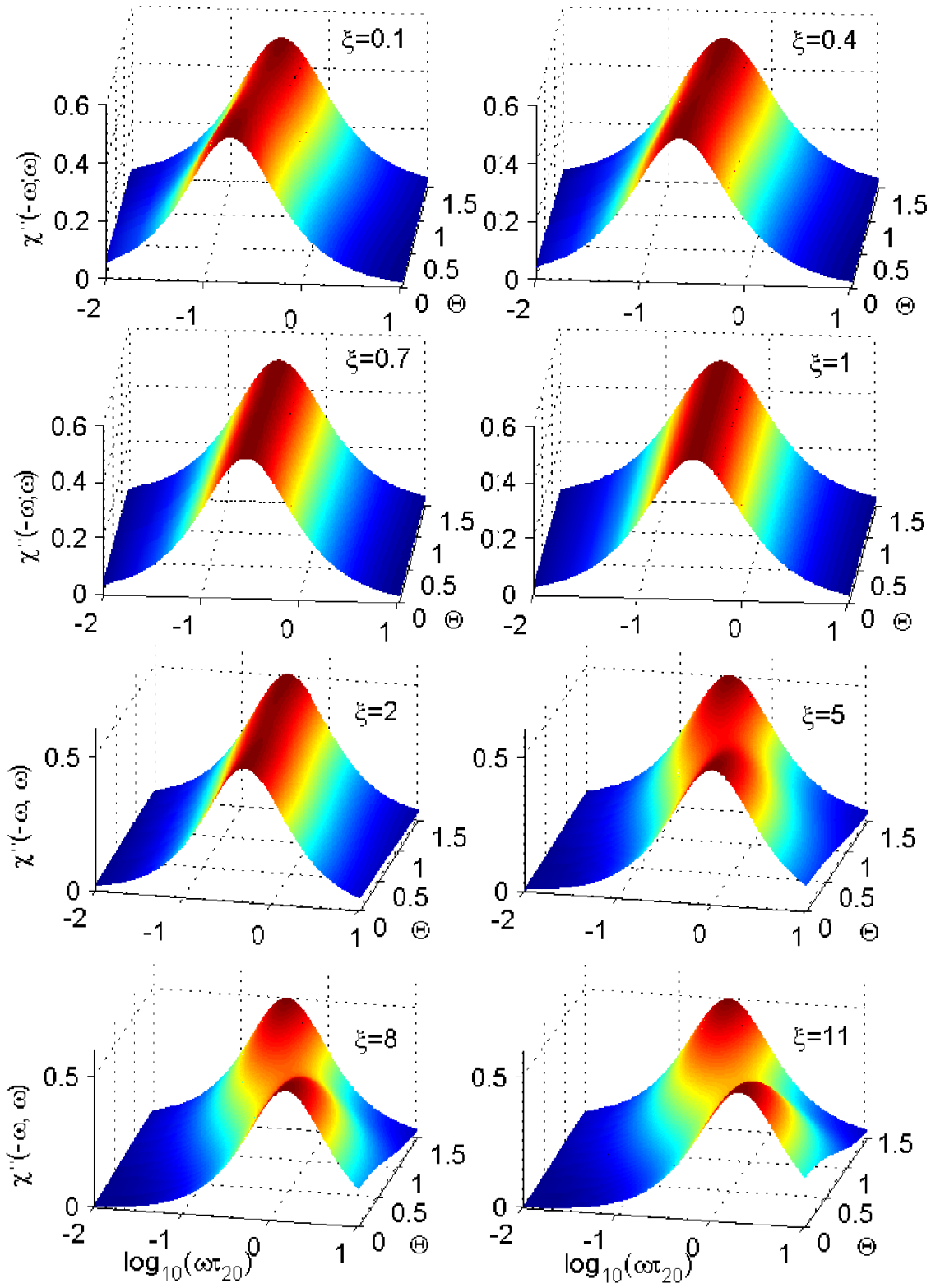

Fig. 4. Dependence of the linear susceptibility $\chi^{\prime \prime}(-\omega ; \omega)$, given by Eq. (32), of the frequency $\omega$ of the ac electric field and of the angle $\Theta$ between permanent dipole moment and the symmetry axis of the molecule - for some values of the anisotropy parameter $\xi$.

comparison with the case $\xi=1$ and $\Theta=0$, especially for $\xi>1$. The frequency of the maximal absorption shifts to lower frequencies with increasing $\xi$ and $\Theta$. It is easy to see, especially in Fig. 5, that the symmetric absorption curves for $\xi=1$ 

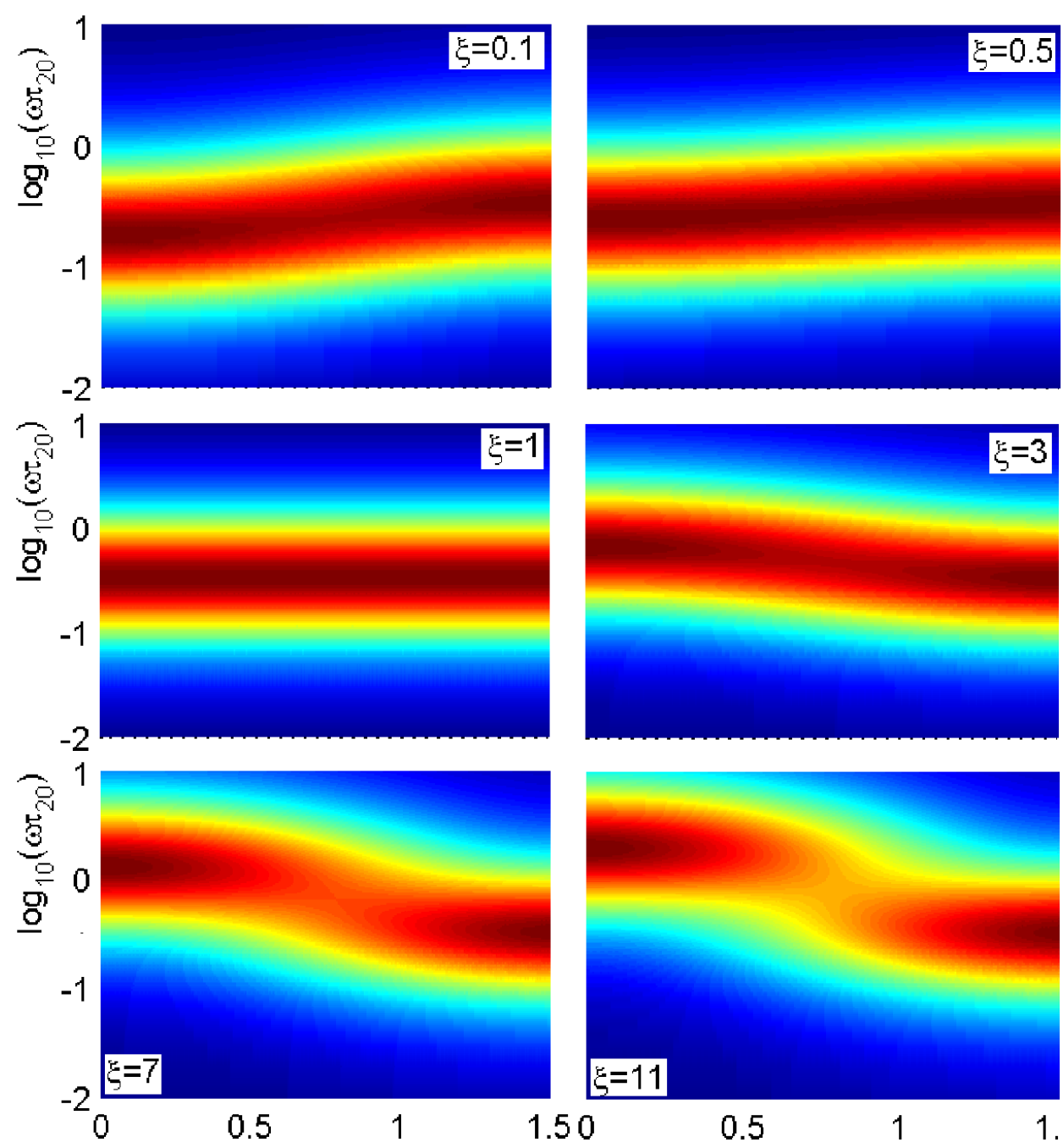

$\Theta$

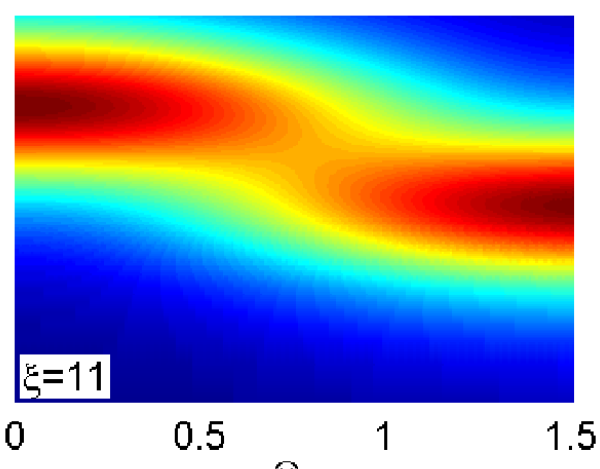

$\Theta$

Fig. 5. Maps - projection of the linear susceptibility $\chi^{\prime \prime}(-\omega ; \omega)$, given by Eq. (32) on the plane $\left(\Theta, \log _{10}\left(\omega \tau_{20}\right)\right)$.

change with the values of $\xi$. For $\xi<1$ we observe a shift of the maximum of absorption towards lower frequencies $\omega$. When the parameter $\xi$ is increased the situation becomes opposite and the effect is much stronger. In the latest picture we also observe a significant decrease in absorption in the region of $\Theta \approx \pi / 4$.

The effect of the nonspherical shape of the molecules is more pronounced in the nonlinear dielectric relaxation. Figure 6 shows the dependences of the nonlinear susceptibility $\chi^{\prime}(-\omega ; \omega, 0,0)$, given by Eq. (39), of the frequency $\omega \tau_{20}$ on the ac electric field, in the logarithmic scale, and on the angle $\Theta$, whereas the 

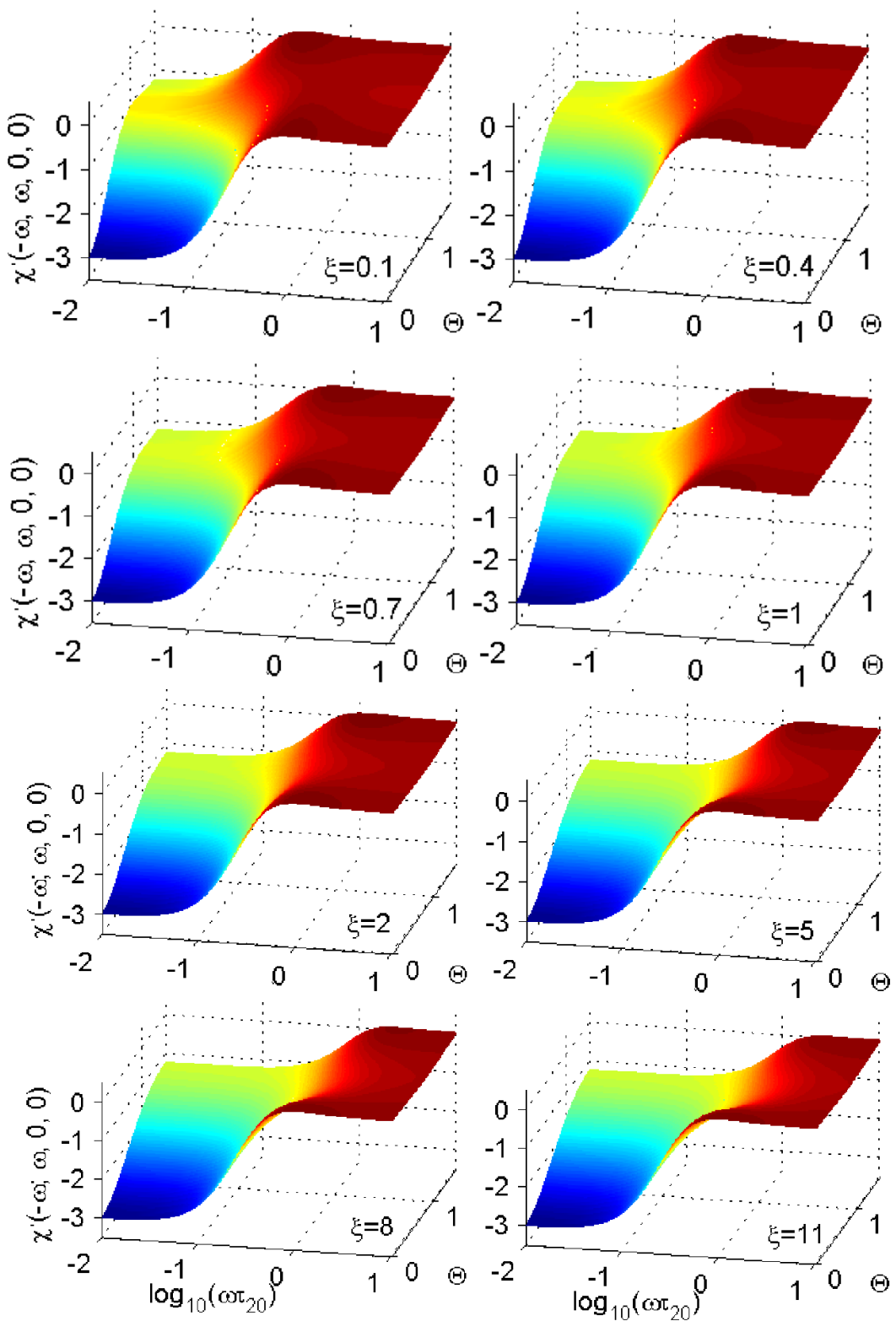

Fig. 6. Dependence of the nonlinear susceptibility $\chi^{\prime}(-\omega ; \omega, 0,0)$, given by Eq. (39), of the frequency $\omega \tau_{20}$ of the ac electric field, in the logarithmic scale, and of the angle $\Theta$ between permanent dipole moment and the symmetry axis of the molecule - for some values of the anisotropy parameter $\xi$.

projection of the nonlinear susceptibility $\chi^{\prime}(-\omega ; \omega, 0,0)$, given by Eq. (39), on the plane $\left(\Theta, \log _{10}\left(\omega \tau_{20}\right)\right)$ is plotted in Fig. 7. The calculations were performed for 


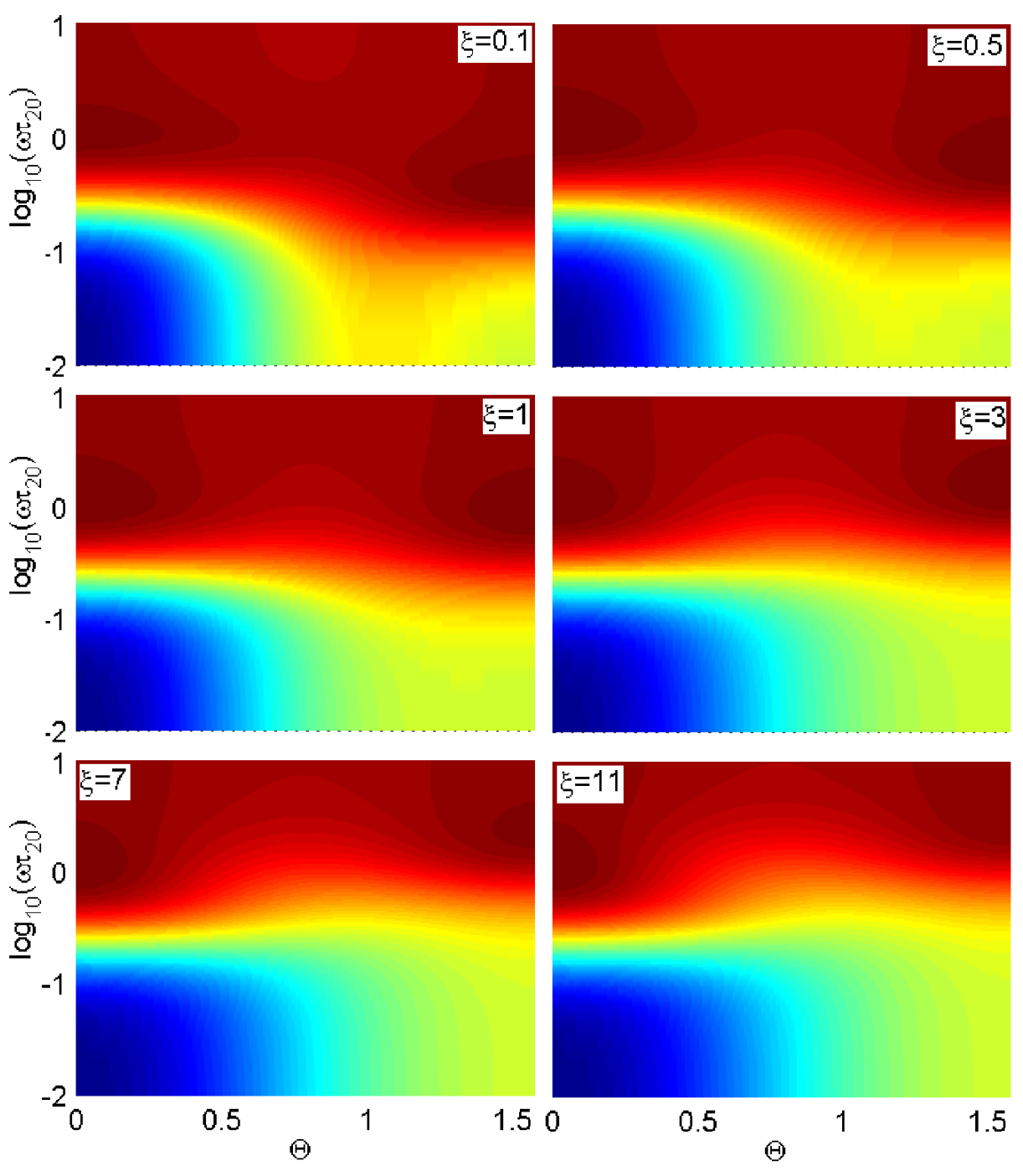

Fig. 7. Maps - projection of the nonlinear susceptibility $\chi^{\prime}(-\omega ; \omega, 0,0)$, given by Eq. (39), on the plane $\left(\Theta, \log _{10}\left(\omega \tau_{20}\right)\right)$.

some values of the anisotropy parameter $\xi$. We see a distinct shift of the region of dispersion towards the lower frequencies with increasing $\Theta$ for all values of the parameter $\xi$. Moreover, the changes in the dispersion for the angle $\Theta>\pi / 4$ are smaller than for $\Theta<\pi / 4$.

Figure 8 shows the dependences of the nonlinear susceptibility $\chi^{\prime \prime}(-\omega ; \omega, 0,0)$, given by Eq. (40), of the frequency $\omega \tau_{20}$ of the ac electric field and of the angle $\Theta$ - for some values of the anisotropy parameter $\xi \leq 1$.

Finally, the projection of the nonlinear susceptibility $\chi^{\prime \prime}(-\omega ; \omega, 0,0)$, given by Eq. $(40)$, on the plane $\left(\Theta, \log _{10}\left(\omega \tau_{20}\right)\right)$ is given in Fig. 9. 

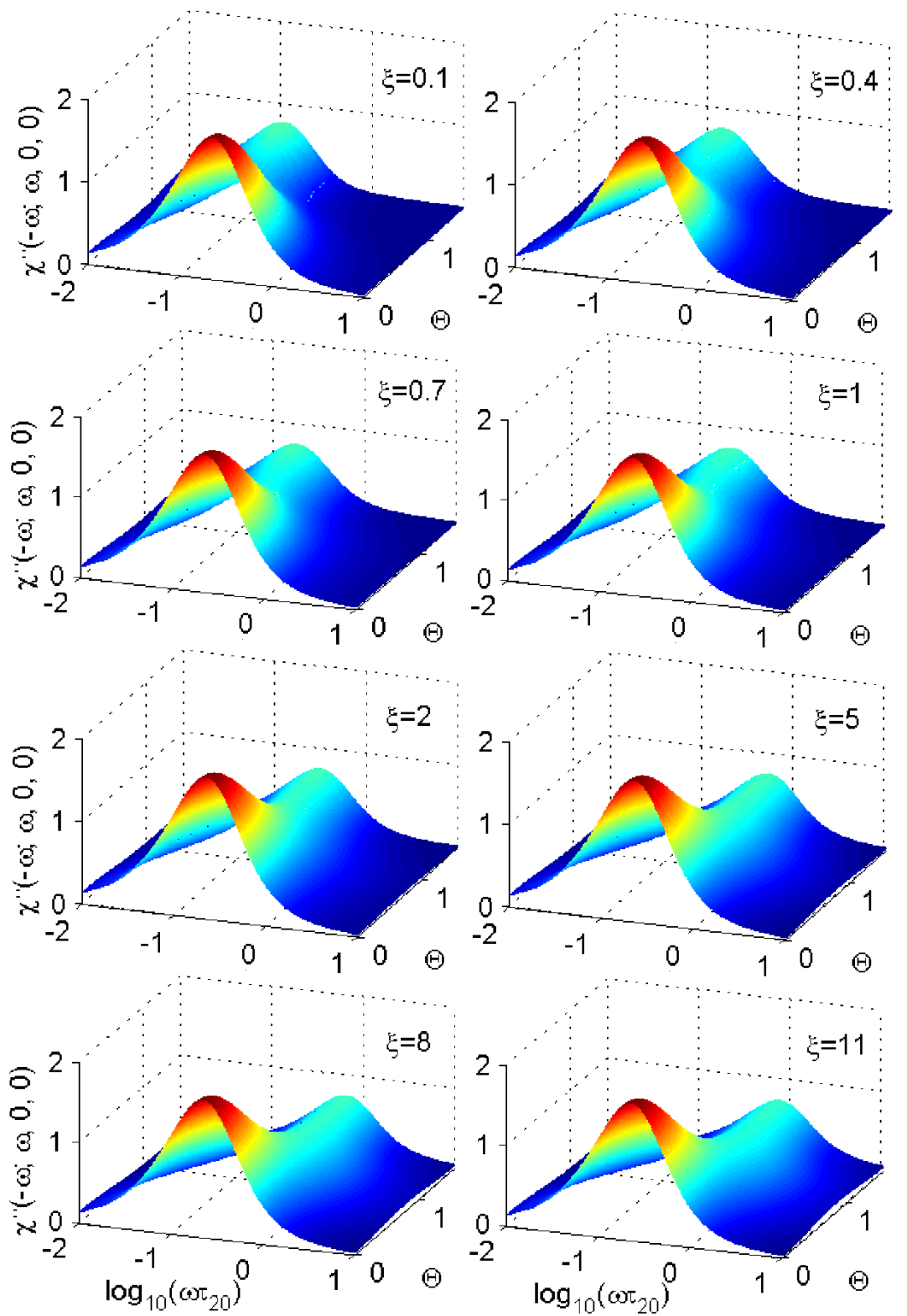

Fig. 8. Dependence of the nonlinear susceptibility $\chi^{\prime \prime}(-\omega ; \omega, 0,0)$, given by Eq. (40), of the frequency $\omega \tau_{20}$ of the ac electric field and of the angle $\Theta$ between permanent dipole moment and the symmetry axis of the molecule — for some values of the anisotropy parameter $\xi$.

Our graphs indicate that the maximum value of nonlinear absorption decreases with increasing $\Theta$ and is shifted towards lower frequencies for $\xi<1$ or 


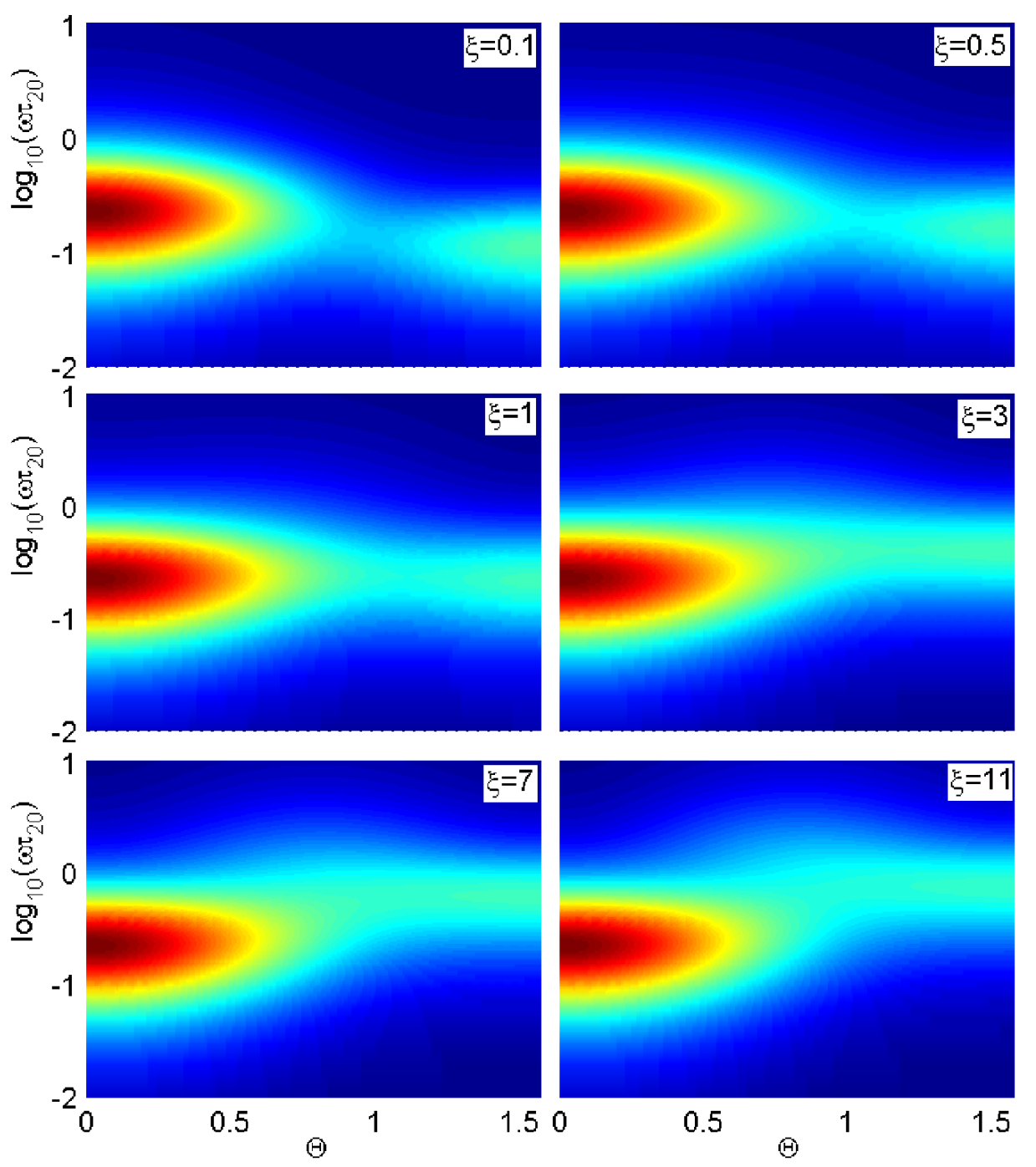

Fig. 9. Maps - projection of the nonlinear susceptibility $\chi^{\prime \prime}(-\omega ; \omega, 0,0)$, given by Eq. (40), on the plane $\left(\Theta, \log _{10}\left(\omega \tau_{20}\right)\right)$.

higher frequencies, when $\xi>1$. For the case of small values of $\xi$ we can observe - Fig. 9 - a small increase in the absorption in the region of $\Theta \approx \pi / 2$, but it disappears when $\xi$ is increased.

For $\Theta=0$ all dispersion and absorption curves are identical with the nonlinear Langevin relaxation, described by the spherical-top rotational diffusion governed by the Smoluchowski-Debye model.

It is our hope that this analysis may be helpful in interpretation of the dielectric relaxation experiments in liquids. 


\section{References}

[1] P. Debye, Polare Molekeln, Hirzel, Leipzig 1929; Polar Molecules, Dover, New York 1945.

[2] F. Perrin, J. Phys. Radium 5, 497 (1934); 7, 1 (1936).

[3] A. Budo, E. Fischer, S. Miyamoto, Phys. Z 40, 337 (1939).

[4] J.L. Dejardin, P.M. Dejardin, Yu.P. Kalmykov, J. Chem. Phys. 107, 508 (1997); 108, 3081 (1998).

[5] J.L. Dejardin, Dynamic Kerr Effect. The Use and Limits of the Smoluchowski Equation and Nonlinear Inertial Responses, World Sci., Singapore 1995.

[6] W.T. Coffey, Yu.P. Kalmykov, J.T. Waldron, The Langevin Equation With Applications in Physics, Chemistry and Electrical Engineering, World Sci., Singapore 1996.

[7] S. Kielich, Nonlinear Molecular Optics, PWN, Warsaw 1977 (in Polish); Nauka, Moscow 1981 (in Russian).

[8] S. Kielich, in: Dielectric and Related Molecular Processes, Ed. M. Davis, Vol. I, Chem. Soc. London 1972, Chap. 7.

[9] B. Kasprowicz-Kielich, S. Kielich, Adv. Mol. Relaxation Processes 7, 275 (1975).

[10] W. Alexiewicz, B. Kasprowicz-Kielich, Adv. Chem. Phys. 85, 1 (1993).

[11] W. Alexiewicz, Mol. Phys. 83, 245 (1994).

[12] A. Piekara, Phys. Z. 28, 671 (1937).

[13] A. Chełkowski, Dielectric Physics, PWN, Warsaw, Elsevier, Amsterdam 1980.

[14] K. De Smet, L. Hellemans, J.F. Rouleau, R. Courteau, T.K. Bose, Phys. Rev. E 57, 1384 (1998).

[15] P. Kȩdziora, J. Jadżyn, K. De Smet, L. Hellemans, Chem. Phys. Lett. 289, 541 (1998).

[16] J. Jadżyn, P. Kȩdziora, L. Hellemans, Phys. Lett. A 251, 49 (1999).

[17] J. Jadżyn, G. Czechowski, D. Bauman, J.L. Dejardin, H. Kresse, R. Douali, Ch. Legrand, Phys. Rev. E 71, 052701 (2005).

[18] J. Jadżyn, G. Czechowski, J.L. Dejardin, M. Ginovska, J. Phys. Chem. A 111, 8325 (2007).

[19] H. Watanabe, A. Morita, Adv. Chem. Phys. 56, 255 (1984).

[20] A. Morita, H. Watanabe, J. Chem. Phys. 77, 1193 (1982).

[21] J.L. Dejardin, J. Mol. Liq. 114, 51 (2004).

[22] W. Alexiewicz, Acta Phys. Pol. B 31, 1051 (2000).

[23] Y.P. Kalmykov, Phys. Rev. E 65, 021101 (2001).

[24] W.T. Coffey, J. Mol. Liquids 114, 5 (2004).

[25] J.L. Dejardin, J. Jadżyn, J. Chem. Phys. 122, 074502 (2005).

[26] J.L. Dejardin, J. Jadżyn, J. Chem. Phys. 123, 174502 (2005).

[27] J.L. Dejardin, J. Jadżyn, J. Chem. Phys. 125, 114503 (2006).

[28] T. Schimomura, Y. Kimura, K. Ito, R. Hayakawa, Colloids Surf. A 148, 155 (1999). 